\title{
Parametric dispersion in electromagnetically induced transparency
}

\author{
M. Müller, ${ }^{1}$ F. Homann, ${ }^{1}$ R.-H. Rinkleff, ${ }^{1}$ A. Wicht, ${ }^{1}$ and K. Danzmann, ${ }^{1,2}$ \\ ${ }^{1}$ Institut für Atom- und Molekülphysik, Abteilung Spektroskopie, Universität Hannover, Callinstrasse 38, D-30167 Hannover, Germany \\ ${ }^{2}$ Max-Planck-Institut für Quantenoptik, Außenstelle Hannover, Callinstrasse 38, D-30167 Hannover, Germany
}

(Received 30 May 2000; published 26 October 2000)

\begin{abstract}
We analyze both experimentally and theoretically the phase shift of a fixed coupling laser field in a $\Lambda$-type system induced by a tunable probe laser field. The measurements are performed with a heterodyne interferometer on a beam of cesium atoms in the $D_{2}$ line. This system exhibits electromagnetically induced transparency with a rapidly varying refractive index. The parametric dispersion of the coupling field over a range of six decades of coupling laser intensities is investigated and found to be in accord with the predictions from the semiclassical model.
\end{abstract}

PACS number(s): 32.70.Jz, 07.57.Pt, 07.57.Ty

Quantum interference in coherently prepared atomic systems can lead to surprising effects like, e.g., inversionless gain or electromagnetic induced transparency (EIT). With a proper choice of fields and atomic states it is possible to design media with certain dispersive and absorptive properties. In previous experiments we have realized transparent media showing negative dispersion [1], just as media with very steep positive dispersion and simultaneously vanishing absorption [2]. For a detailed discussion of these new media the reader is referred to the review articles $[3,4]$.

The dispersive properties of EIT have been studied in many publications, e.g., [5-7], and these properties have been used in a variety of experiments, such as quantum nondemolition measurements [8], experiments with Volkswagen-speed light [9-11], and investigations of optical resonators containing highly dispersive media [12]. However, it is, to our knowledge, the first time that the influence of a probing field frequency onto the phase of a fixed coupling field in a three-level system is experimentally investigated. The observed phase shift of the coupling field depends parametrically on the probe field frequency, and it will be shown that the polarizations of the medium at the probe and the coupling field frequency show totally different spectra under a variation of the probe field frequency.

We are using a heterodyne detection to measure the phase shift of the coupling laser relative to an off-resonant reference laser. This heterodyne interferometer is less sensitive to acoustic and vibrational noise than a homodyne MachZehnder interferometer [2] and always achieves in comparison to frequency modulation spectroscopy (FMS) a modulation index of unity. We demonstrate a reasonable signal-tonoise ratio for our phase measurements, even for coupling laser powers of $1.9 \mathrm{nW}$ in the interaction region corresponding to a field intensity of $12 \mathrm{nW} / \mathrm{cm}^{2}$.

For the theoretical modeling we are using a semiclassical approach in the density-matrix formalism. Denoting the eigenstates of the unperturbed Hamiltonian with $|n\rangle, n$ $=a, b, c$ (Fig. 1) and using the standard rotating-wave approximation, the density-matrix elements in the interaction picture are

$$
\begin{aligned}
& \dot{\rho}_{c c}=\gamma_{0} / 2-\gamma_{0} \rho_{c c}+\gamma_{a c} \rho_{a a}+i\left(\Omega_{a c} \rho_{c a}-\Omega_{c a} \rho_{a c}\right), \\
& \dot{\rho}_{b b}=\gamma_{0} / 2-\gamma_{0} \rho_{b b}+\gamma_{a b} \rho_{a a}+i\left(\Omega_{a b} \rho_{b a}-\Omega_{b a} \rho_{a b}\right),
\end{aligned}
$$

$$
\begin{gathered}
\dot{\rho}_{a a}=-\left(\gamma_{0}+\gamma_{a b}+\gamma_{a c}\right) \rho_{a a}-i\left(\Omega_{a b} \rho_{b a}-\Omega_{b a} \rho_{a b}\right) \\
-i\left(\Omega_{a c} \rho_{c a}-\Omega_{c a} \rho_{a c}\right), \\
\dot{\rho}_{a b}=-\Gamma_{a b} \rho_{a b}-i \Omega_{a b}\left(\rho_{b b}-\rho_{a a}\right)-i \Omega_{a c} \rho_{c b}, \\
\dot{\rho}_{a c}=-\Gamma_{a c} \rho_{a c}-i \Omega_{a c}\left(\rho_{c c}-\rho_{a a}\right)-i \Omega_{a b} \rho_{b c}, \\
\dot{\rho}_{b c}=-\Gamma_{b c} \rho_{c b}+i \Omega_{a c} \rho_{b a}-i \Omega_{b a} \rho_{a c},
\end{gathered}
$$

where

$$
\begin{gathered}
\Gamma_{a b}=\frac{1}{2}\left(2 \gamma_{0}+\gamma_{a b}+\gamma_{a c}\right)+i \Delta_{a b}, \\
\Gamma_{a c}=\frac{1}{2}\left(2 \gamma_{0}+\gamma_{a b}+\gamma_{a c}\right)+i \Delta_{a c}, \\
\Gamma_{b c}=\gamma_{0}-i \delta .
\end{gathered}
$$

$\Delta_{a b}=\omega_{\text {probe }}-\left(\omega_{a}-\omega_{b}\right)$ and $\Delta_{a c}=\omega_{\text {coupl }}-\left(\omega_{a}-\omega_{c}\right)$ are the one-photon detunings of the probe field $\omega_{\text {probe }}$ and the coupling field $\omega_{\text {coupl }}$ from the respective atomic transition frequencies $\omega_{a}-\omega_{b}$ and $\omega_{a}-\omega_{c}$, and $\delta$ is the two-photon detuning $\Delta_{a b}-\Delta_{a c}$. The Rabi frequencies $\Omega_{a i}, i=b, c$ are defined as $\Omega_{a b / c}=\mu_{a b / c} E_{0}^{\text {probe/coupl }} /(2 \hbar)$, where $E_{0}^{j}$ is the amplitude of the electric field $E_{j}(r, t)=\operatorname{Re}\left[E_{0}^{j} \exp \left(i \omega_{j} t\right.\right.$ $\left.\left.-\vec{k}_{j} \cdot \vec{r}\right)\right]$ and $\vec{k}_{j}$ the wave vector for the field at frequency $\omega_{j}$ ( $j=$ probe,coupl). The dipole matrix elements are denoted by $\mu_{a b}$ and $\mu_{a c} \cdot \gamma_{0}$ is the transient loss rate that is derived from the time it takes for an atom to travel through the laser field. This model is well suited for atomic beams, where the ground-state coherence lifetime is much longer than the traveling time. Although there are analytical steady-state solutions for this system that were described first in [13], the simplest way of illustrating the occurring coherence effects is by numerical simulation.

Figure 2 shows the numerical steady-state solutions for the coherences $\rho_{a b}$ and $\rho_{a c}$. The real part of $\rho_{a b}$ shows the normal dispersive shape with a narrow inverted dispersive structure in the center. We want to focus on the fact that the real part of $\rho_{a c}$ has a different shape and includes only the sharp dispersion profile at the two-photon resonance, while the imaginary part of the coupling coherence shows the same EIT behavior as the probe coherence $\rho_{a b}$ [Figs. 2(B), (D)]. Clearly this is no violation of the Kramers-Kronig relation because the parameter that is varied is the frequency of the 


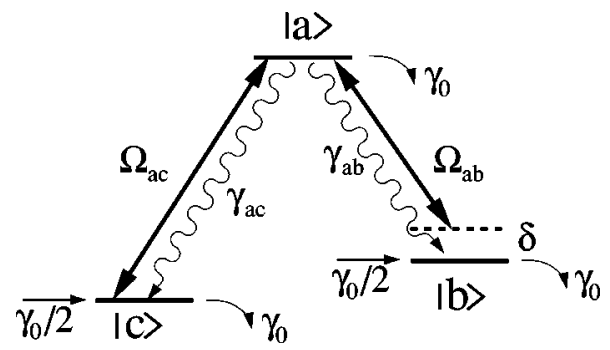

FIG. 1. $\Lambda$-type three-level system. $|i\rangle$, eigenstates of the unperturbed Hamiltonian; $\gamma_{a b}, \gamma_{a c}$, spontaneous emission rates; $\gamma_{0} / 2$, injection rate; $\gamma_{0}$, transient loss.

probe laser, while the coupling laser frequency stays resonant with the coupling transition. Hence we call this behavior parametric dispersion.

If we assume a short interaction length and a low optical density we can relate the matrix elements with our experimentally accessible quantities $d n(\delta)$ and $\alpha(\delta)$ as follows:

$$
\begin{gathered}
\frac{\operatorname{Re}\left(\rho_{a c}\right)}{\Omega_{a c}}=\frac{\hbar}{2 \pi N \mu_{a c}^{2}} d n(\delta), \\
\frac{\operatorname{Im}\left(\rho_{a c}\right)}{\Omega_{a c}}=\frac{\hbar c}{2 \pi N \omega_{a c} \mu_{a c}^{2}} \alpha(\delta) .
\end{gathered}
$$

Here is $d n(\delta)$ the deviation of the refractive index $n(\delta)$ from unity, $\alpha(\delta)$ the absorption coefficient, and $N$ the atomic density. The resulting phase shift is $d \varphi(\delta)$ $=d n(\delta) \omega_{\text {coupl }} l / c$, with $l$ as interaction length.
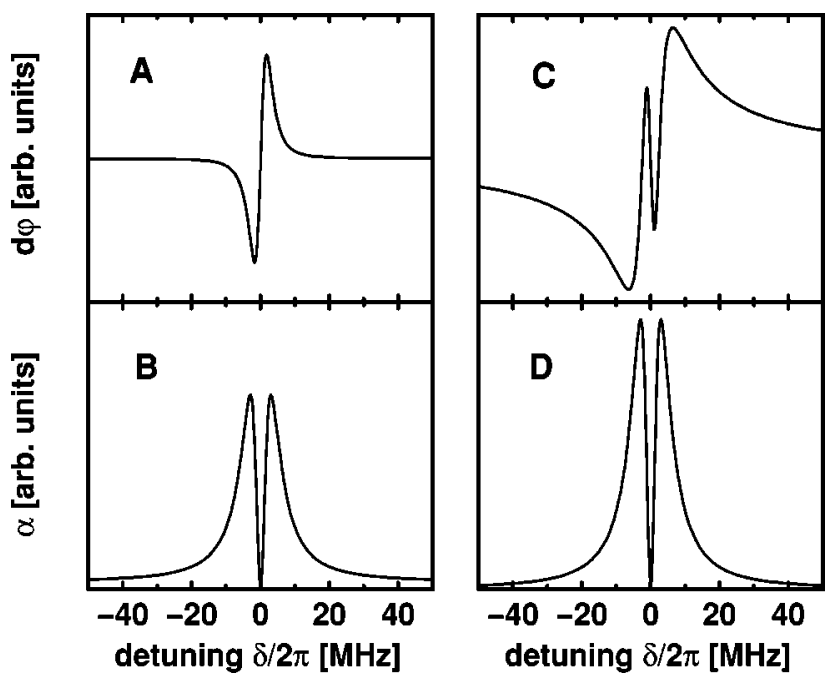

FIG. 2. Theoretical spectra of the parametric phase shift $d \varphi$ (A) and the corresponding absorption $\alpha$ (B). In (C) and (D) phase shift and absorption of the probe field are shown. Here are $\gamma_{0}$ $=10^{5} \mathrm{~s}^{-1}, \quad \gamma_{a b}=2 \pi 2.18 \mathrm{MHz}, \quad \gamma_{a c}=2 \pi 3.05 \mathrm{MHz}, \quad \Omega_{a b}=\Omega_{a c}$ $=0.3 \gamma, \Delta_{a c}=0$.

The derivative of the real part of $\rho_{a c}$ over the coupling Rabi frequency gives a measure for the dispersion at the coupling field frequency. This quantity is also proportional to the reduction of the group velocity for a propagating electromagnetic pulse.

From the steady-state solution of the master equation we get the dispersion of the coupling field at the two-photon resonance:

$$
\frac{\partial}{\partial \delta}\left(\frac{\operatorname{Re}\left(\rho_{a c}\right)}{\Omega_{a c}}\right)=\frac{4 \Omega_{a c}^{2}\left\{\gamma_{0}\left(\gamma+\gamma_{0}\right)\left(\gamma+2 \gamma_{0}\right)\left(\gamma+3 \gamma_{0}\right)+\left[4 \gamma_{a c}\left(\gamma+4 \gamma_{0}\right)+6 \gamma_{0}^{2}\right] \Omega_{a b}^{2}+\left[4\left(\gamma_{a b}+\gamma_{0}\right)\left(\gamma+2 \gamma_{0}\right)-2 \gamma_{0}^{2}\right] \Omega_{a c}^{2}\right\}}{\left(2 \gamma_{0}+\gamma\right)\left[2 \gamma_{0}^{2}+\gamma \gamma_{0}+2 \widetilde{\Omega}^{2}\right]^{2}\left[\gamma_{0}\left(\gamma+\gamma_{0}\right)\left(\gamma+2 \gamma_{0}\right)+\left(4 \gamma_{a c}+8 \gamma_{0}\right) \Omega_{a b}^{2}+\left(4 \gamma_{a b}+8 \gamma_{0}\right) \Omega_{a c}^{2}\right]},
$$

with $\widetilde{\Omega}^{2}=\Omega_{a b}^{2}+\Omega_{a c}^{2}$ and $\gamma=\gamma_{a b}+\gamma_{a c}$. We will consider a small decay rate of the ground-state polarization $\gamma_{0} \ll \gamma$. With this approximation the parametric dispersion in Eq. (3) reduces to the simple form

$$
n^{\prime} \approx C\left(\frac{2 \Omega_{a b}}{\gamma_{0} \gamma+2 \widetilde{\Omega}^{2}}\right)^{2}
$$

where $n^{\prime}$ is the derivative of the refractive index with respect to $\delta$ and $C=2 \pi N \mu_{a c}^{2} / \hbar$ collects the constants from Eq. (2). For low coupling field intensities the dispersion becomes stationary at $n_{\text {sat }}^{\prime} \approx C\left[2 \Omega_{a b} /\left(\gamma_{0} \gamma+2 \Omega_{a b}^{2}\right)\right]^{2}$. The maximum achievable dispersion is thus $C\left(2 \gamma_{0} \gamma\right)^{-1}$ at a probe Rabi frequency of $\Omega_{a b}=\sqrt{\gamma_{0} \gamma / 2}$, where the lifetime of the ground-state coherence $\gamma_{0}$ in our case is given by the interaction time. In principle the ground-state coherence can be very long lived using special techniques $[10,14]$. The above described behavior of the parametric dispersion is plotted in Fig. 3.

The experiments were performed on a Cs atomic beam. We measured the phase shift and the absorption of the coupling beam and the probe beam absorption simultaneously. The experimental setup is sketched in Fig. 4. In these experiments we are using three self-injection-locked diode lasers with spectral bandwidths of $\approx 100 \mathrm{kHz}$ at time scales of milliseconds. The coupling laser was stabilized to the $6 s_{1 / 2}, F$ $=3 \rightarrow 6 p_{3 / 2}, F^{\prime}=4$ transition via polarization spectroscopy. The vacuum wavelength is $852 \mathrm{~nm}$ and the lifetime of the excited level is $30.5 \mathrm{~ns}$ [15], corresponding to a total decay rate $\gamma$ of $2 \pi 5.22 \mathrm{MHz}$ and the optical transition rates $\gamma_{a b}$ $=2 \pi 2.18 \mathrm{MHz}$ and $\gamma_{a c}=2 \pi 3.05 \mathrm{MHz}$.

The reference laser was phase locked to the coupling laser with an offset frequency of $\nu_{\text {off }} \approx 1 \mathrm{GHz}$ to lower frequen- 


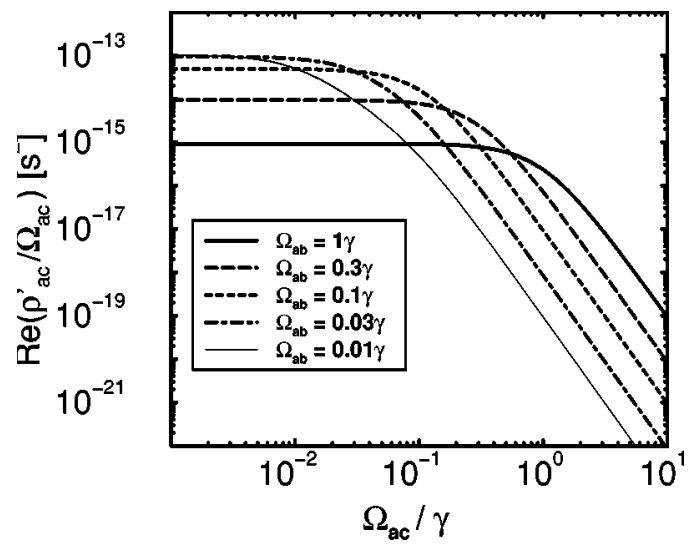

FIG. 3. Calculated dispersion at the coupling field frequency for various probe field intensities with $\gamma=2 \pi 5.22 \mathrm{MHz}$ and $\gamma_{0}$ $=10^{5} \mathrm{~s}^{-1}$. The low power limit has a maximum of $\operatorname{Re}\left(\rho_{a c}^{\prime} / \Omega_{a c}\right)$ $=1.510^{-13} \mathrm{~s}^{-2}$ at $\Omega_{a b}=0.04 \gamma$.

cies. We have chosen a high offset to ensure that there is no influence of the reference field on the sample. The reference offset frequency was tunable to compensate for any phase offsets due to unbalanced arm lengths.

The probe laser was then phase locked to the reference with a $\delta / 2 \pi+\nu_{\text {off }}+9.192631770 \mathrm{GHz}$ offset that corresponds to the reference offset plus the hyperfine splitting in the ground state of cesium plus the two-photon detuning. The offset frequency was provided by a tunable microwave oscillator. The technique of optical phase locking was described before in, e.g., [2]. The beat signals for the phaselocked loops and the reference arm were detected with fast fiber coupled $p-i-n$ photodetectors, and the signal in the probe arm with a $1-\mathrm{GHz} p-i-n$ photoreceiver.

Coupling and probe field had different polarizations and were separated after the interaction zone. The laser intensities were adjusted with discrete metal-coated neutral density filters mounted in a filter wheel to provide good reproducibility.

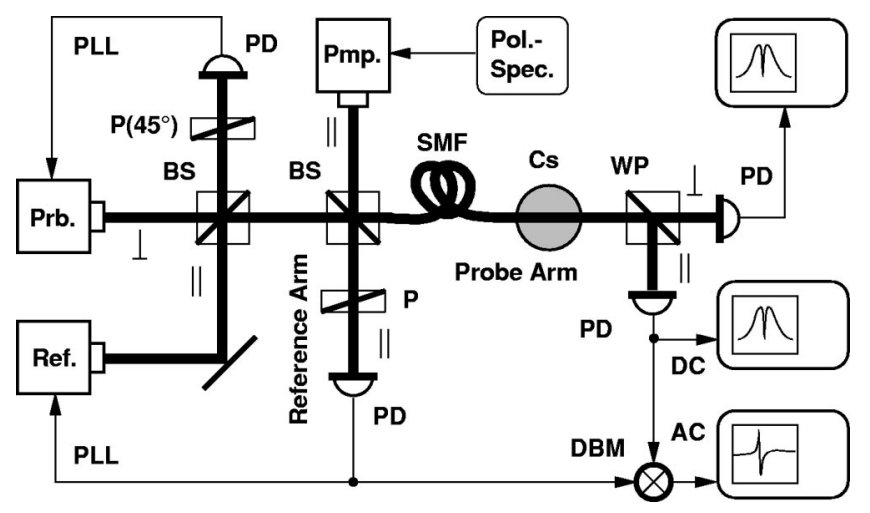

FIG. 4. Experimental setup. Prb., Pmp., and Ref. probe, coupling, and reference laser; $\|$, parallel polarization; $\perp$, vertical polarization; BS, power beam splitter; $\mathrm{P}$, polarizer; $\mathrm{P}\left(45^{\circ}\right)$, polarizer under $45^{\circ}$ with respect to the incident polarization; WP, Wollaston prism; Cs, cesium atomic beam; SMF, single-mode fiber; PD, photodiodes; DBM, double balanced mixer; PLL, optical phase-locked loop.
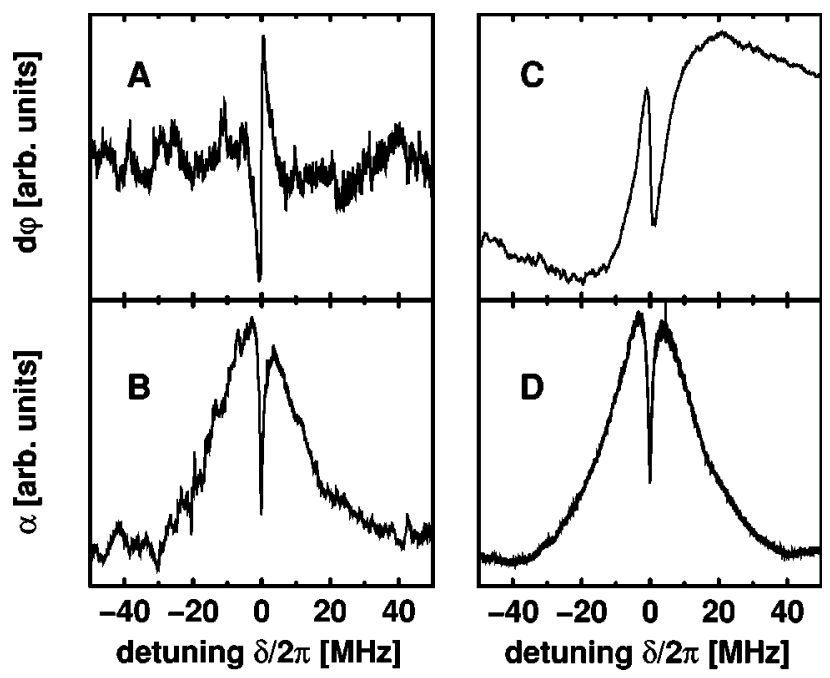

FIG. 5. Typical observed spectra of the parametric phase shift $d \varphi$ (A) and the corresponding absorption $\alpha$ (B). In (C) and (D) phase shift and absorption of the probe field are shown. Probe and coupling laser intensity were $2.5 \mathrm{~mW} / \mathrm{cm}^{2}$.

The three laser fields were superposed at a beam splitter. From one output of the beam splitter the fields propagated to the interaction zone through a single-mode fiber that served as a mode cleaner and ensured a perfect mode matching of the three beams. After the fiber the beams are collimated with a lens doublet that produces a beam waist in the interaction zone of $2200 \mu \mathrm{m}$. The beams passed the sample and were separated by a Wollaston prism. The interference of the reference laser field with the coupling laser field on the photodetectors at the ends of both probe and reference arm generates beat signals oscillating with the difference frequency between these fields. While phases and amplitudes in the reference arm remain constant, the phase and amplitude of the coupling field depend on the absorption and dispersion in the sample. The absorption can be measured directly with the dc photocurrent of the photoreceiver:

$$
I_{\mathrm{dc}}(\delta) \propto\left(E_{0}^{\mathrm{coupl}}\right)^{2} e^{-2 \alpha(\delta) l}+\left(E_{0}^{\mathrm{ref}}\right)^{2} .
$$

The phase shift was obtained by demodulating the ac signal with the beat signal from the reference arm. The amplified beat signals from reference and probe arm are the inputs of the double balanced mixer, and the mixer output signal carries the information about the phase shift of the coupling laser:

$$
I_{\text {mix }}(\delta) \propto\left(E_{0}^{\text {coupl }} E_{0}^{\mathrm{ref}}\right)^{2} e^{-\alpha(\delta) l} \sin [d \varphi(\delta)-\phi],
$$

with $\phi=2 \pi \nu_{\text {off }}\left(L_{\text {probe }}-L_{\text {ref }}\right) / c$, where $L_{\text {probe/ref }}$ are the arm lengths of the interferometer. This residual phase shift $\phi$ is adjusted to zero by the appropriate choice of the reference frequency. The mixer output signal is low pass filtered (20 $\mathrm{Hz}, 18 \mathrm{~dB}$ /octave).

The sample was a cesium atomic beam $(N=2$ $\times 10^{8}$ atoms $/ \mathrm{cm}^{3}$ ) with a residual Doppler linewidth of 4.5 $\mathrm{MHz}$ for the optical transition and $120 \mathrm{~Hz}$ for the two-photon resonance. The length of the interaction region was $5 \mathrm{~mm}$. 


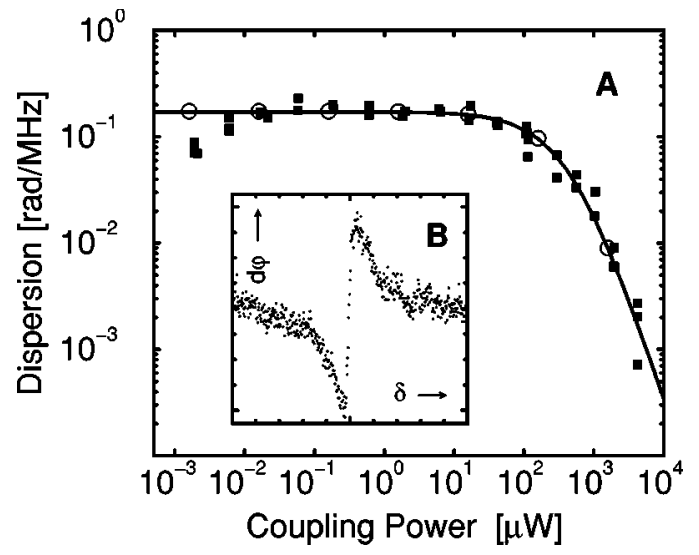

FIG. 6. A, observed parametric phase shift of the coupling field with probe laser intensity $3.1 \mathrm{~mW} / \mathrm{cm}^{2}$. The solid curve shows the semiclassical solution from Eq. (3). The circles show the approximated solution for $\gamma_{0}=10^{5} \mathrm{~s}^{-1} \ll \gamma$ from Eq. (4). B, Measured phase shift of the coupling beam over a span of $10 \mathrm{MHz}$, with a coupling beam intensity of $8.3 \mathrm{nW} / \mathrm{cm}^{2}$. The maximum phase shift is $\pm 37 \mathrm{mrad}$.

Six coils in the three spatial directions were mounted around the interaction region outside the vacuum chamber to compensate for magnetic fields.

The reference offset frequency was adjusted so that the off-resonant phase difference between probe and reference arm canceled. During each scan the coupling laser was fixed to the $6 s_{1 / 2}, F=3 \rightarrow 6 p_{3 / 2}, F^{\prime}=4$ transition, while the probe laser was tuned $100 \mathrm{MHz}$ over the $6 s_{1 / 2}, F=4 \rightarrow 6 p_{3 / 2}, F^{\prime}$ $=4$ transition. A typical spectrum of the parametric dispersion (A) and absorption (B) of the coupling field is shown in Fig. 5. In graphs (C) and (D) the corresponding spectra for the probe field are plotted.

The intensity of the probe beam was fixed at $95 \mu \mathrm{W}$ and the coupling field power was varied over six orders of magnitude from $4.2 \mathrm{~mW}$ down to $1.9 \mathrm{nW}$. The experimental data were fitted to the theoretical spectrum of the phase shift. The derivatives of the phase shift on the two-photon resonance obtained from these fits are plotted in Fig. 6(A). The low power limit, for $d \varphi^{\prime}$, we get is $0.16 \mathrm{rad} / \mathrm{MHz}$. Figure 6(B) demonstrates the good performance of the heterodyne method for $1.3-\mathrm{nW}$ and $420-\mu \mathrm{W}$ coupling and probe field intensity, respectively.

In conclusion, we demonstrated experimentally the parametric phase shift of the coupling field in an EIT scheme. The maximum dispersion in these experiments was limited by saturation broadening of the two-photon resonance. At lower probe laser powers the parametric dispersion is limited by the ground-state relaxation rate.

We have shown that the heterodyne technique is well suited to measure small phase shifts even at low intensities. The observed behavior of the parametric dispersion on the dark resonance confirms the theoretical model used in this work.

This work was supported by the Sonderforschungsbereich SFB 407 of the Deutsche Forschungsgemeinschaft DFG. The authors wish to thank A. Matsko for helpful discussions.
[1] A. Wicht, M. Müller, A. Rocco, R.-H. Rinkleff, and K. Danzmann, Opt. Commun. 179, 107 (2000).

[2] G. Müller, A. Wicht, R.-H. Rinkleff, and K. Danzmann, Opt. Commun. 127, 37 (1996).

[3] E. Arimondo, in Progress in Optics, edited by E. Wolf (Elsevier, Amsterdam, 1996), Vol. 35, pp. 257-354.

[4] S. E. Harris, Phys. Today 50 (7), 36 (1997).

[5] S. E. Harris et al., Phys. Rev. A 46, R29 (1992).

[6] M. Xiao et al., Phys. Rev. Lett. 74, 666 (1995).

[7] A. M. Akulshin et al., Phys. Rev. Lett. 83, 4277 (1999).
[8] A. Sinatra et al., Phys. Rev. A 57, 2980 (1998).

[9] L. V. Hau et al., Nature (London) 397, 594 (1999).

[10] M. M. Kash et al., Phys. Rev. Lett. 82, 5229 (1999).

[11] D. Budker et al., Phys. Rev. Lett. 83, 1767 (1999).

[12] G. Müller, M. Müller, A. Wicht, R.-H. Rinkleff, and K. Danzmann, Phys. Rev. A 56, 2385 (1997).

[13] G. Orriols, Nuovo Cimento Soc. Ital. Fis., B 53, 1 (1979).

[14] D. Budker et al., Phys. Rev. Lett. 81, 5788 (1998).

[15] R. J. Rafac et al., Phys. Rev. A 50, R1976 (1994). 